\title{
Physiotherapy in a sea of change
}

The New Zealand health system is about to embark on its biggest reform in 20 years. This comes on the back of a global pandemic, the like of which has not been seen since 1918. To state we are living in a state of flux is an understatement.

However, within a pandemic, there lies an opportunity. Physiotherapy as a profession within New Zealand has celebrated its centenary as a profession and more recently the centenary of legislation pertaining to the profession. It is a profession that has stayed the course. It has responded to the needs of the time, be it a polio epidemic, or world wars. With the advent of a social insurance scheme (the Accident Compensation Corporation), it has led the world in many respects with regard to direct access and in being an established component of the broader concept of primary care.

Yet, I wonder if there is still a lurking concern that it remains in many ways separate, and not fully integrated as part of the New Zealand health system. Herein lies the opportunity of the era of crisis in which we find ourselves. The role I inhabit in the Ministry of Health is the Chief Allied Health Professions Officer. This is the first time there has been such a role in the Ministry, to work alongside the Chief Medical Officer and Chief Nursing Officer as part of the Executive Leadership team. The establishment of the role stems directly from Dr Ashley Bloomfield as the Director-General of Health, who, as a previous Chief Executive of a District Health Board always had a full clinical team to advise and give direction. As such, he was quick to establish a similar role within the Ministry.

Having worked in the District Health Board environment for an extended period of time, transitioning to the Ministry has been one of the steepest learning curves in my professional career, in learning that the term 'machinery of government' is a real thing. As a Ministry, there is the dual role we play being kaitiaki or steward of the system and working with the sector itself, while also fulfilling the role of public servant.

I say this as a background to my earlier point as to whether there is still that lurking concern that physiotherapy is not seen as fully integrated into the New Zealand health system. While my role is to fulfil the kaitiaki role to the more than 40 professions that fall under the umbrella of allied health, there is the responsibility to identify opportunities where we can achieve better outcomes as we strive for the concept of Pae Ora (Healthy Futures), where we have a population that lives longer, lives better, with equity of outcomes for Māori and all other peoples.

Herein lies the opportunity. Embracing the concept of Pae Ora, we have the opportunity to ask some questions about where the profession is now, and where it could be. The benefit of the profession as a first point of call for musculoskeletal injuries has been well demonstrated. The impact of treating musculoskeletal injuries has a positive impact, not only on physical health, but also on mental health. So this should continue. But what are the opportunities that have not yet been fully embraced? How can physiotherapy as a profession begin to much more consciously align itself to the concept of Pae Ora? How can the profession encompass the fullness of the person and their environment as they move towards a broader realisation of wellness?

While purposefully posed as questions, they aim to represent some of the opportunities that lie before us in this period of change. For many, the impact of changes may be minimal as the revenue stream for services will be uninterrupted. However, there is the potential to begin to look at what else could be, and potentially, most importantly, to look at how physiotherapy better meeting the concept of Pae Ora can be funded. This would be a success of the upcoming reforms.

All of this loops back to a key learning for me personally in gaining a fuller understanding that the Ministry of Health should not be seen as separate to the delivery of health services. Rather, it should be seen as a component of the New Zealand healthcare system. The challenge I believe lies in front of all of us is to be informed of the changes that are occurring, and how physiotherapy as a profession can continue and, if anything, be more aligned to the needs of the population of Aotearoa New Zealand. These are both challenging and exciting times.

\author{
Martin Chadwick DHS, MHS \\ Chief Allied Health Professions Officer, Ministry of Health
}

Email: Martin.Chadwick@health.govt.nz

https://doi.org/10.15619/NZJP/49.2.01 